\title{
SUSY transformations with complex factorization constants. Application to spectral singularities
}

\author{
Boris F. Samsonov \\ Physics Department, Tomsk State University, 36 Lenin Avenue, 634050 Tomsk, \\ Russia \\ E-mail: samsonov@phys.tsu.ru
}

\begin{abstract}
Supersymmetric (SUSY) transformation operators with complex factorization constants are analyzed as operators acting in the Hilbert space of functions square integrable on the positive semiaxis. Obtained results are applied to Hamiltonians possessing spectral singularities which are non-Hermitian SUSY partners of selfadjoint operators. A new regularization procedure for the resolution of the identity operator in terms of continuous biorthonormal set of the non-Hermitian Hamiltonian eigenfunctions is proposed. It is also argued that, if the binorm of continuous spectrum eigenfunctions is interpreted in the same way as the norm of similar functions in the usual Hermitian case, then one can state that the function corresponding to a spectral singularity has zero binorm.
\end{abstract}

Introduction. In many cases the spectrum of non-selfadjoint operators (e.g. Hamiltonians) differ from the spectrum of selfadjoint ones by two essential features. These are (i) the possible presence of exceptional points and (ii) the possible presence of spectral singularities. Note that exceptional points appear already in the finite dimensional case (matrices) whereas spectral singularities are a characteristic feature of Hamiltonians possessing a continuous spectrum and, hence, they are impossible for finite dimensional operators. We think that just by this reason non-selfadjoint operators with spectral singularities are studied in much less details.

Recently, one can notice a growing interest to Hamiltonians possessing a real spectrum and spectral singularities [1, 2, 3, 4. Probably it is due to a remark that they may produce a resonance-like effect in some experiments [2]. One can notice a contradiction in recently published results. In particular, some authors claim that for a Hamiltonian possessing a spectral singularity no any resolution of the identity operator is possible [1, 2]. From the other hand there is a thorough analysis of an exactly solvable complex potential defined on the real axis where the authors prove that there always exists a set of test functions for which the resolution of the identity operator over a biorthonormal set of eigenfunctions takes place [3].

Note also that, as shown in [5], the method of supersymmetric quantum mechanics (SUSY QM, for a review see e.g. [6]) may be very useful for studying both exceptional points and spectral singularities since non-Hermitian Hamiltonians possessing these 
features may appear as SUSY partners of Hermitian Hamiltonians. Another property of non-Hermitian Hamiltonians widely discussed in the current literature is a possible real character of their spectrum (for a review see [7]). Below we will consider only nonHermitian Hamiltonians with a real spectrum which are SUSY partners of Hermitian Hamiltonians. This possibility is open after the publication of papers [8] where the authors showed that SUSY transformations may produce non-Hermitian Hamiltonians with real spectra.

It happens that for a problem on a semiaxis, exceptional points appear if the Jost solution with a real momentum is taken as the transformation function (see below). If we displace this real momentum from the real axis to the complex plane, the transformation function will correspond to a complex factorization constant but the spectral singularity disappears from the spectrum of the non-Hermitian Hamiltonian. In such a way one may realize a smooth path in the space of parameters, the non-Hermitian Hamiltonian $H$ depends on, leading from points where no any spectral singularity is present to a point where $H$ possesses a spectral singularity. This opens a way for regularizing the resolution of the identity operator expressed in terms of continuous spectrum eigenfunctions of $H$ in the point where the spectral singularity is present. To study this possibility in details, one has to consider the Hamiltonian in the vicinity of the spectral singularity in the space of parameters where no problems with the resolution of the identity operator is present and then consider a smooth limit to the singular point.

To realize the above described procedure one needs to use SUSY transformations with complex factorization constants. Therefore we start with a thorough analysis of these transformations as operators acting in the Hilbert space of square integrable functions defined on the positive semiaxis.

Below we will show that a singularity inside the integral describing the resolution of the identity operator is caused by a vanishing bi-normalization coefficient of corresponding continuous spectrum eigenfunction. Therefore, to regularize this resolution, it is sufficiently to shift the factorization constant by an infinitesimal value from the real axis to the complex plane in the normalization factor of continuous spectrum eigenfunctions. Moreover, below we explain what we mean by the binormalization coefficient of the continuous spectrum eigenfunction and arrive at the conclusion that the function corresponding to the spectral singularity has zero binorm.

SUSY transformations of a real scattering potential with complex factorization constants. Let we are given a Hermitian scattering Hamiltonian $h_{0}$ with a real valued potential $v_{0}(x)=v_{0}^{*}(x)$

$$
h_{0}=h_{0}^{*}=-\partial_{x}^{2}+v_{0}(x), \quad x \in \mathcal{R}_{+}:=[0, \infty) .
$$

It is initially defined on the domain $\mathcal{D}_{h_{0}} \subset \mathcal{L}^{2}$ in the space $\mathcal{L}^{2}:=\mathcal{L}^{2}\left(\mathcal{R}_{+}\right)$of functions square integrable on the non-negative semiaxis $\mathcal{R}_{+}$. As the domain $\mathcal{D}_{h_{0}}$ one can choose a set of finite twice continuously differentiable functions $\psi(x)$ with the Dirichlet boundary condition at the origin, $\psi(0)=0$. In this case the closure of $h_{0}$ is self-adjoint in $\mathcal{L}^{2}$ (see, e.g., 9] ) which we will denote by $\bar{h}_{0}=\bar{h}_{0}^{\dagger}$ and its domain by $\mathcal{D}_{\bar{h}_{0}}$. 
For simplicity we will assume also that $h_{0}$ has a purely continuous spectrum,

$$
h_{0} \psi_{k}=E_{k} \psi_{k}, E_{k}=k^{2}, k \geq 0 .
$$

Functions $\psi_{k}(x)$ (generalized eigenfunctions, see e.g. [10]) satisfy the Dirichlet boundary condition at the origin, $\psi_{k}(0)=0$, and asymptotic condition at $x=\infty$. We would like to emphasize that they are assumed to be real valued,

$$
\psi_{k}^{*}(x)=\psi_{k}(x), \quad k \geq 0 .
$$

Moreover, they form an orthonormal basis (in the sense of distributions) in the space $\mathcal{L}^{2}$

$$
\left\langle\psi_{k} \mid \psi_{k^{\prime}}\right\rangle:=\int_{0}^{\infty} d x \psi_{k}^{*}(x) \psi_{k^{\prime}}(x)=\left(k-k^{\prime}\right), \quad \int_{0}^{\infty} d k\left|\psi_{k}\right\rangle\left\langle\psi_{k}\right|=\mathbf{1}
$$

and realize the spectral decomposition of $h_{0}$ which is just the colsure $\bar{h}_{0}$ of the differential operator $h_{0}$ introduced above,

$$
\bar{h}_{0}=\int_{0}^{\infty} d k k^{2}\left|\psi_{k}\right\rangle\left\langle\psi_{k}\right| .
$$

Note that $\psi_{k}(x) \notin \mathcal{L}^{2}$ but they belong to a wider space of functions defining regular functionals over a set of test functions. The action of the operator $h_{0}$ is naturally extended to the space of these functionals. The interested reader may find more information about rigged Hilbert spaces in [10.

Operator (5) is defined on a wider domain $\mathcal{D}_{\bar{h}_{0}}$ than $h_{0}$. It consists of all functions $\psi \in \mathcal{L}^{2}$ such that

$$
\left\|\bar{h}_{0} \psi\right\|^{2}:=\left\langle\bar{h}_{0} \psi \mid \bar{h}_{0} \psi\right\rangle=\int_{0}^{\infty} d k k^{4}\left\langle\psi \mid \psi_{k}\right\rangle\left\langle\psi_{k} \mid \psi\right\rangle<\infty .
$$

Operator $\bar{h}_{0}$ presents the minimal closed and self-adjoint extension of $h_{0}$.

Operator (11) and a non-Hermitian Hamiltonian

$$
H=-\partial_{x}^{2}+V(x)
$$

together with its adjoint $H^{\dagger}=-\partial_{x}^{2}+V^{*}(x)$ are related via intertwining relations

$$
L h_{0}=H L, \quad h_{0} L^{\dagger}=L^{\dagger} H^{\dagger} .
$$

Using the real valued character of $v_{0}$ we obtain a complex conjugate form of equations (7)

$$
L^{*} h_{0}=H^{*} L^{*}, \quad h_{0} L^{* \dagger}=L^{* \dagger} H^{* \dagger}=L^{* \dagger} H .
$$

Here we used the property $H^{*}=H^{\dagger}$ which we assume to hold. We would like to emphasize that $L^{\dagger}$ here should be understood as formally (Laplace) adjoint to $L$. Below we will describe domains of the operators $L, L^{*}$ and $L^{\dagger}$ as differential operators acting in the space $\mathcal{L}^{2}$ and give their closed extensions.

In the simplest case, that we shall consider below, operator $L$ is a first order differential operator

$$
L=-\partial_{x}+w
$$


with $w=w(x)=[\log u(x)]^{\prime}$ being a complex valued superpotential defined with the help of a complex valued solution $u=u(x) \neq 0 \forall x \in(0, \infty)$ of the differential equation $h_{0} u=\alpha u$ with $\alpha$ being, in general, a complex factorization constant. The constant $\alpha$ participates at the factorization of the Hamiltonians $h_{0}$ and $H$

$$
L^{* \dagger} L=h_{0}-\alpha, \quad L L^{* \dagger}=H-\alpha .
$$

Complex conjugate form of these relations may also be useful

$$
L^{\dagger} L^{*}=h_{0}-\alpha^{*}, \quad L^{*} L^{\dagger}=H^{\dagger}-\alpha^{*} .
$$

Potential $V(x)$, defining Hamiltonian (6), is expressed via the superpotential in the usual way, $V(x)=v_{0}(x)-2 w^{\prime}(x)$.

Any solution $\varphi$ of the differential equation

$$
H \varphi_{E}=E \varphi_{E}, \quad E \neq \alpha
$$

may be obtained by the action on a solution of the equation $h_{0} \psi_{E}=E \psi_{E}$ with the operator $L, \varphi_{E}=L \psi_{E}$. When $E=\alpha$ the function $\varphi_{\alpha}=1 / u$ is a solution to equation (12), $H \varphi_{\alpha}=\alpha \varphi_{\alpha}$. Another solution to this equation at $E=\alpha, \widetilde{\varphi}_{a}$, linearly independent with $\varphi_{\alpha}$, may be obtained from the equation $\varphi_{a}(x) \widetilde{\varphi}_{\alpha}^{\prime}(x)-\varphi_{a}^{\prime}(x) \widetilde{\varphi}_{\alpha}(x)=1$.

In general, the transformation $L$ violates boundary conditions. The problem is simplified essentially if $L$ transforms the eigenfunctions of the Hamiltonian $h_{0}, \psi_{k}$, to the eigenfunctions of the Hamiltonian $H, \varphi_{k}$. To guaranty this property we will solve the boundary value problem defined by the equation (12) and the boundary condition

$$
\left[\varphi_{E}^{\prime}(x)+\varphi_{E}(x) w(x)\right]_{x=0}=0 .
$$

The initial domain $\mathcal{D}_{H}$ of the operator $H$ as an operator acting in the space $\mathcal{L}^{2}$ consists of a set of finite twice continuously differentiable functions $\varphi(x)$, satisfying condition (13). The domain $\mathcal{D}_{H^{\dagger}}$ is defined as $\mathcal{D}_{H^{\dagger}}=\left(\mathcal{D}_{H}\right)^{*}$. Note that $H^{\dagger}$ defined on $\mathcal{D}_{H^{\dagger}}$ is not adjoint to $H$ in the sense of the inner product. Below we will give their closed extensions where this property will take place.

Equations (10) and (11) are derived at the level of differential expressions for all the operators involved in these expressions. Operators $h_{0}, H$ and $H^{\dagger}$ have initial domains in the space $\mathcal{L}^{2}$ described above. Using formulas (10) and (11) we can define $L, L^{*}$ and $L^{* \dagger}$ as operators acting in the space $\mathcal{L}^{2}$. Evidently, operator $L(9)$ is well defined on $\mathcal{D}_{h_{0}}$. Therefore we take $\mathcal{D}_{h_{0}}$ as an initial domain for $L$ acting in $\mathcal{L}^{2}$. Similarly, $\mathcal{D}_{H}$ and $\mathcal{D}_{H^{\dagger}}$ may be taken as initial domains for $L^{* \dagger}$ and $L^{\dagger}$ respectively.

The functions $\widetilde{\varphi}_{k}=L \psi_{k}, k \geq 0$ solve the equation $H \widetilde{\varphi}_{k}=k^{2} \widetilde{\varphi}_{k}$, satisfy the condition (13), and the asymptotic condition at infinity and, hence, they are continuous spectrum eigenfunctions for $H$. Similarly to $\psi_{k}$ they do not belong to the space $\mathcal{L}^{2}$ but to a wider space of functions defining regular functionals over a set of test functions. Similar to $h_{0}$ the operator $H$ is extended to the space of these functionals. Note also that applying $L$ to $\psi_{k} \notin \mathcal{L}^{2}$ we naturally extend the action of the transformation operator to the space of functionals. Below we will show that the functions $\varphi_{k}$ participate at the resolution of the identity operator. 
The function $\varphi_{\alpha}=1 / u$ which is a solution to Eq. (12), satisfies the condition (13). Therefore to have the spectrum of the operator $H$ real for a complex $\alpha=-a^{2}$ with $\Im(\alpha) \neq 0$, we have to choose the function $u(x)$ such that $1 / u(x) \rightarrow \infty$ as $x \rightarrow \infty$. This means that a good choice for $u(x)$ is the Jost solution $f(k, x)$ for the Hamiltonian $h_{0}$, $h_{0} f(k, x)=k^{2} f(k, x)$ which has the asymptotics $f(k, x) \rightarrow \exp (i k x)$ as $x \rightarrow \infty$ at a fixed value of $k=-i a, a \in \mathbb{C}$. Thus,

$$
u(x)=f(-i a, x) \rightarrow \exp (a x), \quad \alpha=-a^{2}, \quad x \rightarrow \infty
$$

with $a=d+i b, b \neq 0$ and $d \leq 0$. For $d=0, u(x)$ oscillates at infinity, i.e., it satisfies the asymptotic boundary condition corresponding to a continuous spectrum eigenfunction. As it will be shown below, just in this case a spectral singularity appears in the spectrum of $H$. For $d<0, u(x) \rightarrow 0$ as $x \rightarrow \infty$ and the Hamiltonian $H$ has no spectral singularities. In both cases the operator $H$ has a purely continuous spectrum, $E=E_{k}=k^{2}, k>0$. Below we will distinguish the case when $H$ has a spectral singularity from that when its spectrum is regular.

Regular case. Consider first the case $d<0$. As it was already mentioned, the functions $L \psi_{k}$, satisfy both the equation (12) and the boundary condition (13). Therefore for $d<0$ the functions

$$
\varphi_{k}=N_{k}^{-1} L \psi_{k}, \quad N_{k}:=\left(k^{2}-\alpha\right)^{1 / 2}
$$

form a continuous biorthonormal basis (in the sense of distributions) in $\mathcal{L}^{2}$,

$$
\left\langle\varphi_{k^{\prime}}^{*} \mid \varphi_{k}\right\rangle=\int_{0}^{\infty} d x \varphi_{k}(x) \varphi_{k^{\prime}}(x)=\left(k-k^{\prime}\right), \quad \int_{0}^{\infty} d k\left|\varphi_{k}\right\rangle\left\langle\varphi_{k}^{*}\right|=\mathbf{1} .
$$

The first property follows from the first factorization relation in (10) and the eigenvalue equation (2). The second property is a characteristic feature of a non-Hermitian Hamiltonian with a purely continuous spectrum without spectral singularities (see e.g. [11, 12]). It may be justified by the following chain of equations

$$
\begin{aligned}
& \int_{0}^{\infty} d k\left|\varphi_{k}\right\rangle\left\langle\varphi_{k}^{*}\right|=L \int_{0}^{\infty} d k \frac{\left|\psi_{k}\right\rangle\left\langle\psi_{k}^{*}\right|}{k^{2}-\alpha}\left(L^{*}\right)^{\dagger}=L\left(h_{0}-\alpha\right)^{-1}\left(L^{*}\right)^{\dagger} \\
& =(H-\alpha)^{-1} L\left(L^{*}\right)^{\dagger}=(H-\alpha)^{-1}(H-\alpha)=\mathbf{1} .
\end{aligned}
$$

Here we used equations (15), (3), spectral decomposition of the resolvent of $h_{0}$, first equation (7) and second equation (10).

Applying operator $L^{* \dagger}$ to (15), using the factorization property (10) once again, and eigenvalue equation (12), one obtains the transformation inverse to (15)

$$
\psi_{k}=N_{k}^{-1} L^{* \dagger} \varphi_{k}
$$

Using the basis (15), we obtain the spectral decomposition of $H$ which we will denote by $\bar{H}$

$$
\bar{H}=\int_{0}^{\infty} d k k^{2}\left|\varphi_{k}\right\rangle\left\langle\varphi_{k}^{*}\right| .
$$


From here one finds the operator adjoint to $\bar{H}$

$$
\bar{H}^{\dagger}=\int_{0}^{\infty} d k k^{2}\left|\varphi_{k}^{*}\right\rangle\left\langle\varphi_{k}\right|
$$

Operator $\bar{H}$ is defined on a wider domain than $H$. It consists of all functions $\varphi \in \mathcal{L}^{2}$ such that

$$
\|\bar{H} \varphi\|^{2}=\langle\bar{H} \varphi \mid \bar{H} \varphi\rangle=\int_{0}^{\infty} d k k^{2} \int_{0}^{\infty} d k^{\prime}{k^{\prime}}^{2}\left\langle\varphi \mid \varphi_{k^{\prime}}^{*}\right\rangle\left\langle\varphi_{k^{\prime}} \mid \varphi_{k}\right\rangle\left\langle\varphi_{k}^{*} \mid \varphi\right\rangle<\infty .
$$

Evidently, operator $\bar{H}$ is densely defined by the relation (19). Therefore $\bar{H}^{\dagger}$ is closed. Moreover, since $\bar{H}=\bar{H}^{\dagger \dagger}$ this means that $\bar{H}$ is closed also (see e.g. [13]). Since $\bar{H} \varphi_{k}=H \varphi_{k}=k^{2} \varphi_{k}$ and $\bar{H}^{\dagger} \varphi_{k}^{*}=H \varphi_{k}^{*}=k^{2} \varphi_{k}^{*}$, operators $\bar{H}$ and $\bar{H}^{\dagger}$ are closed extensions of differential operators $H$ and $H^{\dagger}$ respectively.

Closure of transformation operators in the space $\mathcal{L}^{2}$ in the regular case. In the space $\mathcal{L}^{2}$ we have two bases. The functions $\psi_{k}$ form an orthogonal basis and $\varphi_{k}$ form a biorthogonal one (in the sense of distributions). Transformation from one basis to the other is realized by operators

$$
U=\int_{0}^{\infty} d k\left|\varphi_{k}\right\rangle\left\langle\psi_{k}\right|, \quad U \psi_{k}=\varphi_{k}
$$

and

$$
\widetilde{U}=\int_{0}^{\infty} d k\left|\psi_{k}\right\rangle\left\langle\varphi_{k}^{*}\right|, \quad \widetilde{U} \varphi_{k}=\psi_{k}
$$

They have the following property $\widetilde{U} U=\mathbf{1}, U \widetilde{U}=\mathbf{1}$. The first relation here is the usual resolution of the identity operator over the orthonormal basis $\psi_{k}$ (see the second equation in (4)) whereas the second relation is nothing but another form of the second equation in (16).

Using these bases one can construct operators

$$
\bar{L}=\int_{0}^{\infty} d k N_{k}\left|\varphi_{k}\right\rangle\left\langle\psi_{k}\left|, \quad \bar{L}^{\dagger}=\int_{0}^{\infty} d k N_{k}^{*}\right| \psi_{k}\right\rangle\left\langle\varphi_{k}\right|
$$

and

$$
\bar{L}^{*^{\dagger}}=\int_{0}^{\infty} d k N_{k}\left|\psi_{k}\right\rangle\left\langle\varphi_{k}^{*}\left|, \quad \bar{L}^{*}=\int_{0}^{\infty} d k N_{k}^{*}\right| \varphi_{k}^{*}\right\rangle\left\langle\psi_{k}\right| .
$$

Moreover, if we introduce the shifted versions of the Hamiltonians $h_{0}$ and $H$,

$g_{0}:=h_{0}-\alpha=\int_{0}^{\infty} d k N_{k}^{2}\left|\psi_{k}\right\rangle\left\langle\psi_{k}\left|, \quad g_{1}:=H-\alpha=\int_{0}^{\infty} d k N_{k}^{2}\right| \varphi_{k}\right\rangle\left\langle\varphi_{k}^{*}\right|$

and their square roots

$$
g_{0}^{1 / 2}=\int_{0}^{\infty} d k N_{k}\left|\psi_{k}\right\rangle\left\langle\psi_{k}\left|, \quad g_{1}^{1 / 2}=\int_{0}^{\infty} d k N_{k}\right| \varphi_{k}\right\rangle\left\langle\varphi_{k}^{*}\right| .
$$

then from (21), (26), (23) and from (22), (25), (24) it follows that

$$
\bar{L}=U g_{0}^{1 / 2}, \quad \bar{L}^{* \dagger}=\widetilde{U} g_{1}^{1 / 2} .
$$

In case when $H$ is Hermitian, these relations reduce to polar decompositions of the transformation operators obtained in [14]. 
From (23) we see that the domain of definition of $\bar{L}$ consists of all functions $\psi \in \mathcal{L}^{2}$ such that

$$
\|\bar{L} \psi\|^{2}=\int_{0}^{\infty} d k N_{k} \int_{0}^{\infty} d k^{\prime} N_{k^{\prime}}^{*}\left\langle\psi \mid \psi_{k^{\prime}}\right\rangle\left\langle\varphi_{k^{\prime}} \mid \varphi_{k}\right\rangle\left\langle\psi_{k} \mid \psi\right\rangle<\infty .
$$

Similarly, from (24) it follows that the domain of definition of $\bar{L}^{* \dagger}$ consists of all functions $\psi \in \mathcal{L}^{2}$ such that

$$
\left\|\bar{L}^{* \dagger} \psi\right\|^{2}=\int_{0}^{\infty} d k\left|N_{k}\right|^{2}\left|\left\langle\psi \mid \varphi_{k}^{*}\right\rangle\right|^{2}<\infty .
$$

Since $\bar{L}=\bar{L}^{\dagger \dagger}$ these operators are closed (see e.g. [13]). Furthermore, it is easy to check that

$$
\bar{L} \psi_{k}=L \psi_{k}=N_{k} \varphi_{k}, \quad \bar{L}^{* \dagger} \varphi_{k}=L^{* \dagger} \varphi_{k}=N_{k} \psi_{k}
$$

meaning that they are closed extensions of the differential transformation operators $L$ an $L^{* \dagger}$.

Spectral singularity. The eigenfunctions $\psi_{k}, k \geq 0$ of $h_{0}$ can be expressed via the Jost solution $f(k, x)$ as (see e.g. [15])

$$
\psi_{k}(x)=\sqrt{\frac{2}{\pi}} \frac{1}{|F(k)|}[F(-k) f(k, x)-F(k) f(-k, x)], \quad k \geq 0
$$

where $F(k)=f(k, 0)$ is the Jost function for $h_{0}$. Since $\bar{h}_{0}$ is self-adjoint, $F(k)$ does not vanish for any real $k \neq 0$, i.e. $F(k) \neq 0 \forall k \in \mathbb{R} \backslash 0$ [15]. From here one finds

$$
L \psi_{k}(x)=\sqrt{\frac{2}{\pi}} \frac{1}{|F(k)|}[F(-k) L f(k, x)-F(k) L f(-k, x)] .
$$

For any factorization constant $\alpha=-a^{2}, a=d+i b$ with $b \neq 0$ and $d<0$, both $L f(k, x) \neq 0$ and $L f(-k, x) \neq 0 \forall k \geq 0$. But for $d=0$ one has $\alpha=-a^{2}=b^{2}>0$ and since $u(x)=f(-i a, x)=f(b, x)$, this implies that $L f(b, x)=0$, but $L f(-b, x) \sim 1 / u(x)$ and hence $L \psi_{k=|b|} \sim 1 / u(x) \rightarrow \exp (-i b x)$ as $x \rightarrow \infty$. Therefore the function $\varphi_{\alpha}(x)=1 / u(x)$ has the asymptotics $\varphi_{\alpha}(x) \rightarrow \exp (-i b x)$ as $x \rightarrow \infty$ (i.e., this is the Jost solution for $H$ at $k=-b$ ) and at the same time, as it was already mentioned, it satisfies the boundary condition (13). By this reason the point $E=k^{2}=b^{2}$ belongs to the continuous spectrum of $H$ and since the corresponding eigenfunction $\varphi_{k=|b|}(x)$ coincides (up to a nonzero constant) with the Jost solution for $H$, the point $E=k^{2}=b^{2}$, according to the definition (see e.g. [12, 4, 5]), is the spectral singularity for $H$. By this reason and by the definition of the Jost function as the value of the Jost solution at the origin, the Jost function for $H$ vanishes at $k=-b$. Furthermore, the resolvent of $H$ grows in $\mathcal{L}^{2}$ norm in the vicinity of $k=-b$ faster than in the absence of the spectral singularity [4] and a singularity appears in the resolution of the identity operator at $k=|b|[12,3]$.

Another remarkable property of the function $L \psi_{|b|}(x)$ is the value of its binorm. First we would like to stress that the notion of norm (or binorm) has a sense if the normalization (or corresponding bi-normalization) integral has a finite value which is 
not the case for the continuous spectrum eigenfunctions. Nevertheless, the usual physical terminology attaches a certain significance to the term "normalization of a continuous spectrum eigenfunction". Namely, according to the first equation (44), one says that the continuous spectrum eigenfunctions are "normalized to the Dirac delta function". Note that the Dirac delta is not a real function but a distribution. Thus, the above statement about the normalization of the continuous spectrum eigenfunction $\psi_{k_{0}}$ should be understood in the sense of distributions as a functional $\left(k-k_{0}\right)$ which, while acting on a test function $\Phi(k)$, gives the result $\Phi\left(k_{0}\right)$. Just in a similar way, taking into account the first formula (16), one can say that the continuous spectrum eigenfunction $\varphi_{k}$ for a Hamiltonian without spectral singularities is "bi-normalized to the Dirac delta function". Let us consider now what happens if the continuous spectrum eigenfunction corresponds to a spectral singularity.

Taking into account factorization (10) and eigenvalue equation (2), one obtains

$$
\left\langle\left(L \psi_{k}\right)^{*} \mid L \psi_{k^{\prime}}\right\rangle=\left\langle\psi_{k}^{*} \mid L^{* \dagger} L \psi_{k^{\prime}}\right\rangle=\left(k^{2}-\alpha\right)\left(k-k^{\prime}\right)
$$

where the use of (10) has been made. Evidently, this quantity vanishes at $k^{2}=\alpha=b^{2}$ and the binorm (29), depending on the sign of $b$, has a first order zero at either $k=b$ or $k=-b$ times the delta-function singularity which reduces the right hand side of (29) to zero since the delta-function belongs to a class of slowly increasing generalized functions. We have to note that the right (and, hence, the left) hand side of (29) should be understood in the sense of distributions. This means that acting by this functional on a test function of $k^{\prime}$, one obtains a function of $k$. If the test function is regular at $k^{\prime 2}=\alpha=b^{2}$, the limit $k^{2} \rightarrow \alpha=b^{2}$ in (29) gives zero. In other words, the limit $k^{2} \rightarrow \alpha=b^{2}$ in (29) is zero functional defined on a set of test functions. In particular, as the set of test functions one can choose the space of continuous square integrable functions $\Phi\left(k^{\prime}\right), k^{\prime}>0$. Actually, the set of test functions is larger but here we leave open the question of how large it is. We have to note that zero functional is regular. This means that it may be presented as an ordinary Lebesgue integral with a function $\mathcal{F}\left(k^{\prime}\right)$ which is zero almost everywhere times a test function. By this reason the value of $\mathcal{F}\left(k^{\prime}=|b|\right)$ does not affect the zero value of the functional. In particular, this function may even be undefined at $k^{\prime}=|b|$ (see an example below). Nevertheless, keeping the interpretation described above for the normalization of the continuous spectrum eigenfunctions, we say that the continuous spectrum eigenfunction corresponding to the spectral singularity has zero binorm. The final comment here is that this result is due to the vanishing normalization factor $N_{k}^{2}=k^{2}-\alpha$ at $k^{2}=b^{2}$ if $\alpha=b^{2}$ present in (29) but not to the value of the bi-normalization integral (16) at $k=k^{\prime}= \pm b$.

Similar equation has been obtained for a non-Hermitian Hamiltonian defined in the Hilbert space of functions square integrable on the whole real line in [3] (see Eq. (11) of that paper). This permits us to hope that the the continuous spectrum eigenfunction corresponding to a spectral singularity of a non-Hermitian Hamiltonian has zero binorm not only for a class of Hamiltonians considered in this article but also for a more general case. 
Resolution of the identity operator for a Hamiltonian with a spectral singularity. For any $d<0$ the functions $\varphi_{k}$ realize the resolution of the identity operator (see the second equation in (16) ) which we rewrite in the coordinate representation

$$
\int_{0}^{\infty} d k \varphi_{k}(x) \varphi_{k}(y)=\int_{0}^{\infty} d k \frac{\left(L \psi_{k}\right)(x)\left(L \psi_{k}\right)(y)}{k^{2}-\alpha}=(x-y) .
$$

For $d=0$ the integrand contains a first order pole at $k^{2}=\alpha=b^{2}$. This is just a characteristic feature of the spectral singularity. In paper [12] a regularization procedure is proposed for guarantying the corresponding resolution of the identity operator. In a recent paper [3], using a particular example, a thorough analysis of a similar regularization is given. Below, using the just established fact that the origin of this pole is the normalization factor for $\varphi_{k}$, we propose another regularization for this integral.

For $d=0\left(\alpha=b^{2}\right)$, instead of the functions $\varphi_{k}$ given in (15), let us consider the functions $\tilde{\varphi}_{k}=\left(k^{2}-b^{2}-i \varepsilon\right)^{-1 / 2} L \psi_{k}$ with the sign of (infinitesimal) $\varepsilon$ possibly depending on the sign of $b$ (see an example below) which differ from $\varphi_{k}$ by the normalization factor only and use them in the left hand (and the middle) part of equation (30). The integrand in this equation is well defined $\forall \varepsilon \gtrless 0$ now, but the right hand side is not the Dirac delta anymore. Using the property that this relation should be understood in the sense of distributions and the known fact (see e.g. [10]) that there always exists a set of test functions such that one can interchange the limit as $\varepsilon \rightarrow \pm 0$ with the sign of the integral, one restores the necessary behavior of the right hand side of (30) taking the limit $\varepsilon \rightarrow \pm 0$ after the integral is calculated. More precisely, we have first to apply the functional at the middle part of (30) to a test function and after that take the limit $\varepsilon \rightarrow \pm 0$. This statement may be additionally justified by the chain of equations

$$
\begin{aligned}
& \int_{0}^{\infty} d k\left|\tilde{\varphi}_{k}\right\rangle\left\langle\tilde{\varphi}_{k}^{*}\right|=L \int_{0}^{\infty} d k \frac{\left|\psi_{k}\right\rangle\left\langle\psi_{k}^{*}\right|}{k^{2}-b^{2}-i \varepsilon}\left(L^{*}\right)^{\dagger}=L\left(h_{0}-b^{2}-i \varepsilon\right)^{-1}\left(L^{*}\right)^{\dagger} \\
& =\left(H-b^{2}-i \varepsilon\right)^{-1} L\left(L^{*}\right)^{\dagger}=\left(H-b^{2}-i \varepsilon\right)^{-1}\left(H-b^{2}\right) \rightarrow \mathbf{1}, \quad \varepsilon \rightarrow \pm 0 .
\end{aligned}
$$

obtained in a similar way as the chain (17). We note that two signs \pm before $\varepsilon$ do not mean that both signs are relevant. Only one sign leads to the desired result but it may depend on the sign of $b$.

We have to note also that the above arguments have no strict mathematical rigor and should be considered as an indication that this property may really take place and probably may rigorously be proven following the lines similar to that which one uses for proving the usual Parseval formula (see e.g. [15]).

We would like also to mention that the above described procedure is a typical method for regularizing divergent integrals (see e.g. [10]) but here we apply it at the level of distributions. Similar possibility for regularizing the resolution of the identity operator at the singular point is also mentioned in [3] where the authors note that the infinitesimal shifting the pole of an integrand from the real axis to the complex plane may be considered as a procedure alternative to a deformation of the integration contour. 
Exactly solvable example. Let us choose $v_{0}(x)=0$. The Jost solution for $h_{0}$ is the simple exponential $f(k, x)=\exp (i k x)$ so that $u(x)=\exp (a x), a \in \mathbb{C}, a \neq 0$, and $\alpha=-a^{2}$. The superpotential is just a constant $w=a$ and the transformed Hamiltonian contains the kinetic energy only, $H=-\partial_{x}^{2}$, but the boundary condition at $x=0$ for equation (12) contains a complex number, $\varphi^{\prime}(0)+a \varphi(0)=0$. We recognize here an example of a non-Hermitian Hamiltonian with a spectral singularity first proposed by Schwartz [16] (see also [4]). The functions $\varphi_{k}$ have the form

$$
\varphi_{k}=\left(k^{2}-\alpha\right)^{-1 / 2} \sqrt{\frac{2}{\pi}}[a \sin (k x)-k \cos (k x)] .
$$

By the direct calculation one finds the value of the integral

$$
\left\langle\left(L \psi_{k}\right)^{*} \mid L \psi_{k^{\prime}}\right\rangle=\left(k^{2}+a^{2}\right)\left(k-k^{\prime}\right)
$$

which vanishes at $k^{2}=-a^{2}=b^{2} \forall k^{\prime} \geq 0$ if $d=0$. Thus, this is zero functional acting on a set of test functions of the variable $k^{\prime}$. The continuous spectrum eigenfunction for $k=|b|$ is $1 / u=\exp (-i b x)$. If we tried to calculate the binorm of $1 / u$ using the first equation (16) for $k=k^{\prime}=|b|$ we will find that this quantity does not exist since the integral of $\exp (-2 i b x)$ over the interval $(0, \infty)$ does not converge. This is just the illustration of the property discussed above that the function defining zero functional in the form of a usual Lebesgue integral is undefined for $k^{\prime}=|b|$. Nevertheless, we say that the continuous spectrum eigenfunction corresponding to the spectral singularity has zero binorm in the sense described above.

Let us calculate now the integral in the left hand side of (30) for $\alpha=b^{2}>0$. For this purpose we shift the parameter $b^{2}$ in the denominator of the function (32) from the real axis to either the upper half or the lower half of the complex plane by an infinitesimal value $\varepsilon, b^{2} \rightarrow b^{2}+i \varepsilon$. We will see that the sign of $\varepsilon$ depends on the sign of $b$. After some simple manipulations with trigonometric functions and up to a term proportional to $\varepsilon^{2}$ in the denominator, one obtains for the integrand in (31) the following approximation

$$
\begin{gathered}
\frac{\pi}{2} \varphi_{k}(x) \varphi_{k}(y) \approx \cos (k x) \cos (k y)+\left[k^{2}+\left(\frac{\varepsilon}{2 b}-i b\right)^{2}\right]^{-1} \\
\times\left[b^{2} \cos k(x+y)-i b k \sin k(x+y)+\frac{i \varepsilon}{2}(\cos k(x+y)+\cos k(x-y))\right] .
\end{gathered}
$$

After integrating this expression with respect to $k$ over the interval $(0, \infty)$, the first term gives just the desired Dirac delta function. Thus, it remains to show that all other terms give a vanishing result as either $\varepsilon \rightarrow+0$ or $\varepsilon \rightarrow-0$. Not that all integrals here are standard provided $b \varepsilon>0$ and arguments of trigonometric functions are positive (see formulas (3.723.2) and (3.723.3) in [17]). These integrals can easily be continued to the case $b \varepsilon<0$ and a negative argument of cosine function,

$$
\int_{0}^{\infty} \frac{\cos a x}{\beta^{2}+x^{2}} d x= \pm \frac{\pi}{2 \beta} e^{\mp|a| \beta}, \quad \int_{0}^{\infty} \frac{x \sin c x}{\beta^{2}+x^{2}} d x=\frac{\pi}{2} e^{\mp c \beta}, \quad c>0 .
$$


Here $\Im a=0$ and the upper sign corresponds to $\Re_{\underline{i}} 0$ and the lower sign to $\Re_{\mathfrak{j}} 0$. Thus the desired integral may be approximated as

$$
\int_{0}^{\infty} d k \varphi_{k}(x) \varphi_{k}(y) \approx(x-y)+\frac{e^{\mp z(x+y)}}{z}\left[ \pm b^{2}-i b z \pm \frac{i \varepsilon}{2}\right] \pm \frac{i \varepsilon}{2 z} e^{\mp z|x-y|}, z:=\frac{\varepsilon}{2 b}-i b
$$

with the upper sign for $b \varepsilon>0$ and the lower sign for $b \varepsilon<0$. Replacing the parameter $z$ in the middle term of the right hand side of this equation by its value, one can see that this term is equal to zero only for $b \varepsilon>0$. For $b \varepsilon<0$ it survives in the limit $\varepsilon \rightarrow 0$. The last term is a regular functional acting on a test function $\Phi(y), y>0$, represented by the usual Lebesgue integral. Splitting the integration interval in this integral with $b \varepsilon>0$ in two parts, $(0, x)$ and $(x, \infty)$, one obtains

$$
\frac{i \varepsilon}{2 z}\left[e^{-z x} \int_{0}^{x} e^{z y} \Phi(y) d y+e^{z x} \int_{x}^{\infty} e^{-z y} \Phi(y) d y\right], \quad \Re(z)>0 .
$$

From here it follows that this is a decreasing function of $x$ provided $\Phi(y) \rightarrow 0$ as $y \rightarrow \infty$ and therefore for $\varepsilon \rightarrow 0$ this term vanishes. Thus, in the limit $\varepsilon \rightarrow 0$ the right hand side of formula (35) reduces to the Dirac delta for a set of continuous test functions vanishing at infinity and the sign of $\varepsilon$ coinciding with the sign of $b$.

Conclusion. In this paper a careful analysis of SUSY transformations with complex factorization constants as operator acting in the Hilbert space of square integrable functions defined on the positive semiaxis is given. Obtained results are applied to non-Hermitian Hamiltonians which are SUSY partners of self-adjoint operators. Using the same arguments, which permit to say that the continuous spectrum eigenfunctions of a Hermitian Hamiltonian are normalized to the Dirac delta function, we arrived at the conclusion, that the continuous spectrum eigenfunction of a nonHermitian Hamiltonian corresponding to the spectral singularity has zero binorm.

It is also shown that a singularity inside the integral describing the resolution of the identity operator is caused by the vanishing bi-normalization factor $\left(k^{2}-\alpha\right)$ of corresponding continuous spectrum eigenfunction with $\alpha=b^{2}>0$ at the singular point $k^{2}=b^{2}$. A new procedure for regularizing the resolution of the identity operator at the singular point is proposed. It consists of an infinitesimal shifting of the SUSY factorization constant from the real axis to the complex plane in the normalization factor of the corresponding scattering function. Obtained results are illustrated by the simplest example of a non-Hermitian Hamiltonian having the kinetic energy only but a complex boundary condition at the origin. Our results, thus, support the conclusion made in [3] that the resolution of the identity operator for a non-Hermitian Hamiltonian possessing a spectral singularity may be constructed in contrast to the statement made in [1, 2].

Despite this positive result, we have to note that, since, as it has been noticed by Lyance [12], there is no possibility for constructing a Hermitian operator equivalent to a non-Hermitian Hamiltonian with a spectral singularity, the relevance of such nonHermitian Hamiltonians as fundamental objects in quantum mechanics is still doubtful. Nevertheless, for a non-Hermitian Hamiltonian nearly having a spectral singularity (and 
having no exceptional points) such a Hermitian operator exists and can be constructed. The scattering cross section for this Hamiltonian has the ordinary physical meaning and it may exhibit a resonance behavior near the singular point of its non-Hermitian counterpart [18].

Acknowledgment. The author is grateful to A. Sokolov and to one of the referees for useful comments. The work is partially supported by Russian Science and Innovations Federal Agency under contract 02.740.11.0238 and Russian Federal Agency of Education under contract P1337.

[1] Longhi S 2009 Phys. Rev. B 80 165125;

Longhi S 2010 Phys. Rev. A 81022102

[2] Mostafazadeh A and Mehri-Dehnavi 2009 J. Phys. A 42 125303;

Mostafazadeh A 2009 Phys. Rev. A 80 032711;

Mostafazadeh A 2009 Phys. Rev. Lett. 102220402

[3] Andrianov A A, Cannata F and Sokolov A V 2010 J. Math. Phys. 51052104

[4] Guseinov G SH 2009 Pramana J. Phys. 73587

[5] Samsonov B F 2005 J. Phys. A 38 L397;

Samsonov B F 2005 J. Phys. A 38 L571

[6] Mielnik B and Rosas-Ortiz O 2004 J. Phys. A 3710007

[7] Bender C M 2007 Rep. Progr. Phys. 70947

[8] Cannata F, Junker G and Trost J 1998 Phys.Lett. A 246 219; Andrianov A, Cannata F, Dedonder J-P and Ioffe M 1999 Int. J. Mod. Phys. A 142675

[9] Kostuchenko A G and Sargsyan I S 1979 Distribution of eigenvalues. Selfadjoint ordinary differential operators (Moscow: Nauka)

[10] Gelfand I M and Shilov G E 1964 Generalized functions 1 (New York: Academic)

[11] Naimark M A 1968 Linear differential operators (New York: Ungar)

[12] Lyance V E 1964 Mat. Sb. 64 521; ibid 6547 (English transl.: 1967 Amer. Math. Soc. Transl. (2) 60185 ; ibid 227)

[13] Debnath L and Mikusiński P 1999 Introduction of Hilbert spaces with applications. (San Diego: Academic)

[14] Samsonov B F 2000 J. Phys. A 33591

[15] Levitan B M 1987 Inverse Sturm-Liouville problems (Utrecht: VNU Science)

[16] Schwartz J 1960 Comm. Pure Appl. Math 13609

[17] Gradshteyn I S and Ryzhik I M 2007 Table of integrals, series, and products (Burlington: Academic)

[18] Samsonov B F 2010 Preprint arXiv 0081776 [quant-ph] 